\title{
Transmission of phonons through grain boundaries In diamond films
}

\author{
D. T. Morelli \\ Physics Department, General Motors Research and Environmental Staff, Warren, Michigan 48090-9055 \\ C. Uher \\ Physics Department, University of Michigan, Ann Arbor, Michigan 48109 \\ C. J. Robinson a) \\ Raytheon Company Research Division, Lexington, Massachusetts 02181
}

(Received 3 November 1992; accepted for publication 4 January 1993)

\begin{abstract}
The influence of grain boundary scattering of lattice waves in high quality diamond films has been determined by the measurement of thermal conductivity down to $0.15 \mathrm{~K}$. We find that the mean free path for lattice vibrations (phonons) exceeds substantially the crystallite size and approaches the physical size of the sample. This implies that a significant transmission of phonons occurs between the grains of these polycrystalline diamond materials.
\end{abstract}

With the advent of new growth techniques, research on the properties of diamond has undergone a rebirth in recent years. The capability of producing very high quality polycrystalline films has greatly enhanced the prospects of exploiting the extreme physical properties of this substance. One area of great interest has been the thermal conductivity $\kappa$ of synthetically produced diamond. Vapordeposited material is now made routinely with room temperature thermal conductivities up to $85 \%$ that of bulk single crystals. It is not yet clear, however, what processes limit the conductivity to values below that of single crystal material. Some time ago, it was recognized ${ }^{1,2}$ that the polycrystalline nature of the film morphology could provide some limitation to the thermal conductivity at and even above room temperature. More recently, detailed studies of the anisotropy of the thermal conductivity ${ }^{3}$ as well as a through-the-thickness gradient in $\kappa^{4,5}$ have provided some indirect evidence of the influence of grain boundary scattering. On the other hand, it has also been shown ${ }^{6}$ that very low levels of defects within the grains, most likely associated with small concentrations of nondiamond carbon, are capable of strongly degrading the thermal conductivity over the temperature range $20-300 \mathrm{~K}$. Separation of the influence of grain boundaries and other defects is complicated by the fact that samples with smaller grains usually have more defects since the latter tend to be concentrated at grain boundaries. ${ }^{7}$ One can, in principle, differentiate to some extent between the effects of intragrain defects and grain boundaries on the heat conduction by measuring $\kappa$ over a wide temperature range. This is due to the different frequency dependences of the scattering processes. Published data to date extend over the temperature range of approximately $4-400 \mathrm{~K}$. Near the upper end of this range, intrinsic phonon-phonon processes begin to limit the conductivity in high quality material. Near the lower end, grain boundary scattering plays a large role; however, other defects still influence the conductivity in this temperature range. In order to determine unequivocally the magnitude of grain boundary scattering, the thermal conductivity

\footnotetext{
a) Presently Visiting Scientist, Massachusetts Institute of Technology, Cambridge, MA 02139.
}

must be measured to still lower temperature to eliminate completely the effect of intragrain defcets. In this letter, we present measurements of $\kappa$ down to $0.15 \mathrm{~K}$ which allow us to determine directly the influence of grain boundaries on the thermal conductivity of vapor-deposited diamond. Surprisingly, we find that the phonon mean free path exceeds the average crystallite size of the samples by roughly an order of magnitude, implying a large transmission coefficient of phonons between grains at low temperatures.

The samples used in this work were fabricated at the Raytheon Company by standard chemical vapor deposition (CVD) processes discussed in the literature. ${ }^{8} \mathrm{We}$ have studied two samples which are characteristic of high quality vapor-deposited diamond: a sample, designated $H$, grown by hot-filament vapor deposition and a second sample designated $M$ and grown using microwave plasma assisted vapor deposition. Both of these samples exhibit a columnar grain structure typical of all CVD material. Near the substrate surface, the grains are a few micrometers in size, while at the top surface, the grain size is in the range $50-100 \mu \mathrm{m}$, yielding an average grain size on the order of $25-50 \mu \mathrm{m}$. Sample M, however, has been polished on both sides so that the average grain size is larger than that of sample $\mathrm{H}$, on the order of $40-70 \mu \mathrm{m}$. Additionally, sample $\mathrm{H}$ exhibits one-phonon absorption in the infrared, ${ }^{9}$ indicating the presence of defects which are most likely associated with nondiamond carbon. The infrared spectrum of sample $\mathbf{M}$, on the other hand, is nearly the same as bulk single crystal diamond. The thermal conductivity of these two samples was previously measured from $7-300 \mathrm{~K} .{ }^{6}$ At room temperature, the thermal conductivity of sample $M$ was $17.8 \mathrm{~W} \mathrm{~cm}^{-1} \mathrm{~K}^{-1}$, while that of sample $\mathrm{H}$ was 11.1 $\mathrm{W} \mathrm{cm}^{-1} \mathrm{~K}^{-1}$.

The thermal conductivity was measured using the steady-state technique from $7 \mathrm{~K}$ down to $0.15 \mathrm{~K}$ by clamping the samples to the mixing chamber of a dilution refrigerator. A small metal film heater was attached to the free end of the sample using thermally conductive epoxy. The temperature difference across the sample was determined by calibrated germanium resistance sensors traceable to an NBS standard. The maximum relative error in the determination of the temperature difference is estimated to be 


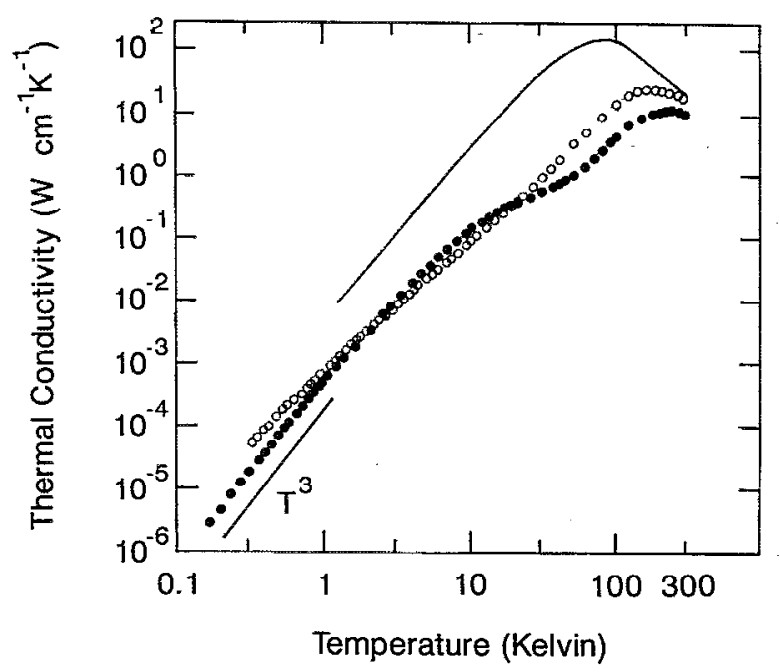

FIG. 1. Thermal conductivity of two diamond films from 0.15 to $300 \mathrm{~K}$. Sample $\mathrm{H}$, closed circles, was grown by hot filament $\mathrm{CVD}$, and sample $\mathrm{M}$, open circles, by microwave assisted CVD. Solid line indicates typical thermal conductivity of single crystal natural diamond.

1\%. The absolute error in the measurement is determined by the geometry of the samples, which are bars of rectangular cross section, and is not more than $5 \%$. The actual sample dimensions were $11.1 \times 3.0 \times 0.5 \mathrm{~mm}^{3}$ and 10.0 $\times 3.0 \times 0.6 \mathrm{~mm}^{3}$, respectively.

Figure 1 shows our results for the conductivity of samples $\mathrm{H}$ and $\mathrm{M}$ below $7 \mathrm{~K}$ combined with earlier measurements above this temperature. The data thus extend over three decades in temperature and six orders in conductivity. As the temperature is lowered below $300 \mathrm{~K}$, both samples exhibit a peak in conductivity, at $170 \mathrm{~K}$ for sample $\mathrm{M}$ and $240 \mathrm{~K}$ for sample $\mathrm{H}$. Below the maximum in $\kappa$ for sample $\mathrm{H}$, a strong depression in the curve centered near $50 \mathrm{~K}$ is observed. Sample $\mathrm{M}$, on the other hand, exhibits a much weaker and broader depression centered near $15 \mathrm{~K}$. Because the dip in sample $M$ occurs at lower temperature than in sample $\mathbf{H}$, the conductivity of the latter sample actually exceeds the former in the range 2-20 K. Below 2 $\mathrm{K}$, the curves cross again and begin to approach the classic $T^{3}$ dependence of diffuse scattering from a boundary.

The dips in the thermal conductivity curves for diamond films have been attributed ${ }^{6}$ to scattering from large scale (as opposed to point) defects of unknown origin. In this picture, the defects scatter phonons independent of frequency $\omega$ for phonons with $\omega>\omega_{c}$ and at a rate proportional to $\omega^{4}$ for phonons with $\omega<\omega_{c}$. The crossover frequency is determined by the spatial extent $D$ of the defect and is given by $\omega_{c}=v / D$, where $v$ is the phonon velocity. The dip in the thermal conductivity curve occurs at a temperature when the dominant phonon frequency equals $\omega_{c}$. This results in a defect size of approximately $5 \mathrm{~A}$ for sample $\mathrm{H}$ and $15 \mathrm{~A}$ for sample $\mathrm{M}$. The combination of this scattering rate with a frequency independent rate due to grain boundary scattering and a standard ${ }^{10}$ phononphonon Umklapp rate provides a very good fit to the data over the range $4-400 \mathrm{~K}^{8}$ Because the dip in the conductivity seems to be larger in samples which contain fairly

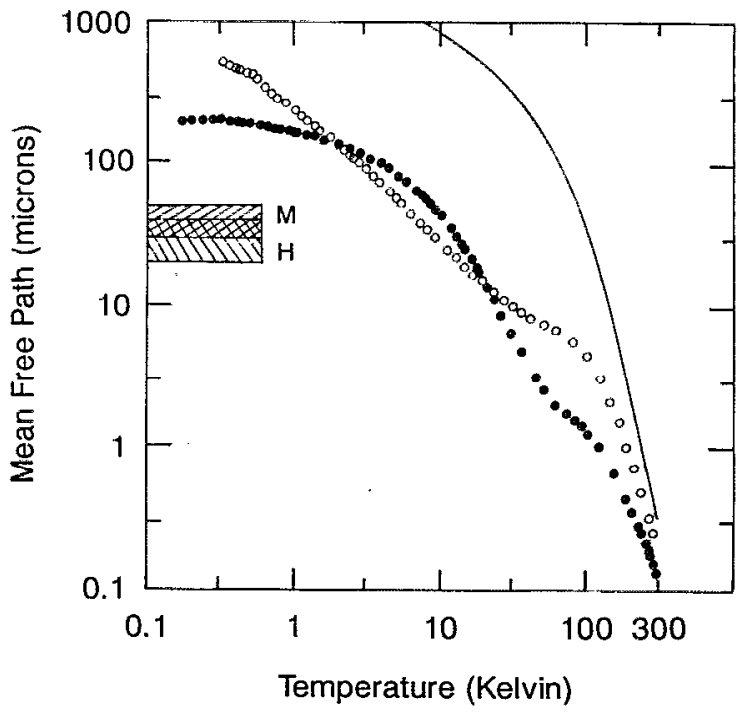

FIG. 2. Phonon mean free path as a function of temperature for samples $\mathbf{H}$ and $\mathbf{M}$. Symbol designation is as in Fig. 1. Hatched areas indicate average crystallite size of samples $H$ and $M$, respectively, and solid line is the phonon mean free path in bulk single crystal natural diamond.

large amounts of $s p^{2}$-bonded carbon, it has been suggested ${ }^{6}$ that these defects may in fact be regions of disordered carbon material at a very low level of concentration. Some support for this picture is provided by recent results on single crystals in which regions of disordered carbon are created by neutron irradiation. ${ }^{11}$

In understanding the present results, more light can be shed on the nature of the conduction process by applying the simple kinetic relation for the heat conductivity:

$$
\kappa=1 / 3 c_{p} \rho v L,
$$

where $c_{p}$ is the specific heat per unit mass, $\rho$ the mass density, and $L$ the phonon mean free path. The validity of this approach in analyzing the thermal conductivity of single crystal and CVD diamond has been discussed previously. ${ }^{12}$ Values of $L$, derived from this formula using the mass density and specific heat ${ }^{13}$ of bulk diamond ${ }^{14}$ and the phonon velocity calculated from the bulk elastic constants $^{15}$ are shown in Fig. 2. The depressions in the conductivity referred to above occurring near 50 and $15 \mathrm{~K}$ for samples $H$ and $M$, respectively, are clearly visible on the monotonically decreasing (with increasing temperature) $L(T)$ curve. What is surprising is the magnitude of the phonon mean free path at low temperatures. Despite the fact that these materials are polycrystalline with grain sizes on the order of $50 \mu \mathrm{m}$, the phonon mean free path approaches $200 \mu \mathrm{m}$ for sample $\mathrm{H}$ and over $500 \mu \mathrm{m}$ for sample $M$. These values are on the order of the sample thickness, indicating that the specimen surface is becoming the predominant scatterer of phonons as the temperature is lowered. This implies that, in these CVD diamond materials, a large transmission of phonons occurs between grains at low temperature.

The above conclusion raises some interesting questions about the conductivity of CVD diamond at temperatures above $1 \mathrm{~K}$, in particular with regard to the "dips" occur- 
ring at 50 and $15 \mathrm{~K}$, respectively, for samples $H$ and $M$. One possibility is that the transmission of phonons across grain boundaries in this material is dependent on the degree of the grains' surface roughness relative to the phonon wavelength, and significantly larger transmission coefficients are obtained when the phonon wavelength exceeds the size of the surface asperities. This would imply surface roughness features of approximately $5 \mathrm{~A}$ for sample $\mathrm{H}$ and $15 \mathrm{~A}$ for sample $\mathrm{M}$. As has already been mentioned, there is strong evidence that defects tend to be concentrated at grain boundaries in CVD diamond. If this is the case, then the present results would be consistent with a phonongrain boundary transmission coefficient dependent on the concentration and spatial extent of disorder at the interface. While thermal conductivity studies cannot pinpoint the nature of the defect, rather only its size and concentration, this scenario is certainly consistent with optical investigations and provides a consistent explanation of the conductivity over the entire investigated temperature range. Since these effects can influence the magnitude of the conductivity at room temperature and even higher, their understanding is crucial to heat transfer applications in which the highest conductivity material is required.

${ }^{1}$ V. I. Nepsha, N. F. Reshetnikov, Yu. A. Klyuev, G. B. Bokii, and Yu. A. Pavlov, Sov. Phys. Dokl. 30, 1275 (1985).
${ }^{2}$ D. T. Morelli, C. P. Beetz, and T. A. Perry, J. Appl. Phys. 64, 3063 (1988).

${ }^{3}$ J. E. Graebner, S. Jin, G. W. Kammlott, B. Bacon, L. Seibles, and W. F. Banholzer, J. Appl. Phys. 71, 5353 (1992).

${ }^{4}$ J. E. Graebner, S. Jin, G. W. Kammlott, J. A. Herb, and C. F. Gardinier, Appl. Phys. Lett. 60, 1576 (1992).

${ }^{5}$ J. E. Graebner, S. Jin, G. W. Kammlott, J. A. Herb, and C. F. Gardinier, Nature 359, 401 (1992).

${ }^{6}$ D. T. Morelli, T. M. Hartnett, and C. J. Robinson, Appl. Phys. Lett. 59, 2112 (1991).

${ }^{7}$ A. V. Heatherington, C. J. H. Wort, and P. Southworth, J. Mater. Res. 5, 1591 (1990).

${ }^{8}$ C. J. Robinson, T. M. Hartnett, R. P. Miller, C. B. Willingham, J. E. Graebner, and D. T. Morelli (unpublished).

${ }^{9}$ C. B. Willingham, T. M. Hartnett, C. J. Robinson, and C. Klein, in Applications of Diamond Films and Related Materials (edited by $\mathrm{Y}$. Tzeng, M. Yoshikawa, M. Murakawa, and A. Feldman (Elsevier, Amsterdam, 1991), p. 157.

${ }^{10}$ D. G. Onn, A. Witek, T. Z. Qiu, T. R. Anthony, and W. F. Banholzer, Phys. Rev. Lett. 68, 2806 (1992).

${ }^{11}$ D. T. Morelli, T. A. Perry, and J. W. Farmer, Phys. Rev. B 47, 131 (1993).

${ }^{12}$ T. R. Anthony, J. L. Fleischer, J. R. Olson, and D. G. Cahill, J. Appl. Phys. 69, 8122 (1991).

${ }^{13}$ Y. S. Touloukian, R. W. Powell, C. Y. Ho, and P. G. Klemens, Thermophysical Properties of Matter (Plenum, New York, 1970), Vol. IV, p. 202.

${ }^{14}$ The specific heat of single crystal diamond is proportional to $T^{3}$ below $100 \mathrm{~K}$, and in the absence of any experimental data, we assume the same behavior of our films.

${ }^{15} \mathrm{~J}$. W. Vandersande, Phys. Rev. B 13, 4560 (1976). 